\title{
Modification of Second-Order Nonlinear IIR Filter for Compensating Linear and Nonlinear Distortions of Electrodynamic Loudspeaker
}

\author{
Kenta Iwai \\ Organization for Research and Development of \\ Innovative Science and Technology, Kansai University, Japan \\ Email: kenta1986@gmail.com
}

\author{
Yoshinobu Kajikawa \\ Faculty of Science and Engineering, \\ Kansai University, Japan \\ Email: kaji@kansai-u.ac.jp
}

\begin{abstract}
In this paper, we propose a modified 2nd-order nonlinear IIR filter for compensation of both linear and nonlinear distortions of electrodynamic loudspeakers. A nonlinear IIR filter is a nonlinear compensator and is based on a physical nonlinear model of electrodynamic loudspeakers. However, it is difficult to compensate nonlinear distortions when a loudspeaker has large sharpness at the lowest resonance frequency, at which the displacement of the diaphragm becomes large. Although it is necessary to compensate the sharpness of the loudspeaker, the nonlinear IIR filter cannot compensate the sharpness because it does not have a linear filtering feature. In this paper, we propose a modified 2nd-order nonlinear IIR filter that can compensate not only the nonlinear distortions but also the sharpness by employing the linear characteristics of the loudspeaker with the desired sharpness. Experimental results show that the proposed filter can compensate the linear and nonlinear distortions more effectively than a conventional filter.
\end{abstract}

\section{INTRODUCTION}

Electrodynamic loudspeakers generate nonlinear distortions, which degrade the sound quality. This is because the nonlinearities of electrodynamic loudspeakers are caused by the large displacement of the diaphragm, particularly around the lowest resonance frequency [1]. One of the methods of compensating nonlinear distortions is to use a mirror filter [2], [3]. This filter is derived from linear and nonlinear differential equations and is designed by a 2nd-order nonlinear IIR filter [4]. This filter considers the nonlinearities of electrodynamic loudspeakers, for example, those of the force factor and stiffness, enabling it to reduce the nonlinear distortions caused by the physical parameters.

A problem with this filter is the difficulty of compensating the nonlinear distortions when the sharpness of the loudspeaker is large, which means that the displacement of the diaphragm becomes large around the lowest resonance frequency. Although it is necessary to compensate the sharpness of the loudspeaker, the 2nd-order nonlinear IIR filter cannot compensate the sharpness because of its compensating principle [5], [6], that is, this filter does not have a linear filtering part. Moreover, the loudspeaker has narrow frequency band for sound reproduction when its resonance frequency is high. Although it is required to move the resonance frequency to lower frequency, the nonlinear IIR filter cannot compensate the resonance frequency for the same reason as noted above.

In this paper, the 2nd-order nonlinear IIR filter is modified so that it can compensate not only nonlinear distortions but also the sharpness. The modified filter employs linear characteristics of the loudspeaker with the desired sharpness.
The linear characteristics of the loudspeaker generally have sharpness and resonance angular frequency. Hence, the proposed filter can compensate not only the sharpness but also the resonance angular frequency.

\section{CONVENTIONAL 2ND-ORDER NONLINEAR IIR FILTER}

The 2nd-order nonlinear IIR filter [4] is a nonlinear digital filter that compensates nonlinear distortions of electrodynamic loudspeakers and is based on the following nonlinear differential equation:

$$
\frac{B l(x) A_{0}}{R_{\mathrm{dc}}} u(t)=m_{0} a(t)+K(x) x(t)+\left\{R_{\mathrm{m}}+\frac{B l^{2}(x)}{R_{\mathrm{dc}}}\right\} v(t),
$$

where $m_{0}$ is the mechanical mass [kg], $K_{0}$ is the stiffness of the damper and suspension $[\mathrm{N} / \mathrm{m}], R_{\mathrm{m}}$ is the equivalent mechanical resistance $[\mathrm{Ns} / \mathrm{m}], R_{\mathrm{dc}}$ is the electrical resistance $[\Omega]$, $B l_{0}$ is the force factor $[\mathrm{Wb} / \mathrm{m}], A_{0}$ is the gain of the analogue part, $u(t)$ is the input voltage [V], $x(t), v(t)=d x(t) / d t$ and $a(t)=d^{2} x(t) / d t^{2}$ are the displacement [m], velocity $[\mathrm{m} / \mathrm{s}]$ and acceleration $\left[\mathrm{m} / \mathrm{s}^{2}\right]$ of the diaphragm and $B l(x)$ and $K(x)$ are the displacement-dependent force factor and stiffness, respectively. $B l(x)$ and $K(x)$ can be represented by polynomial functions as [1]:

$$
\begin{aligned}
B l(x) & =B l_{0} b(x)=B l_{0}\left(1+b_{1} x+b_{2} x^{2}\right), \\
K(x) & =K_{0} k(x)=K_{0}\left(1+k_{1} x+k_{2} x^{2}\right),
\end{aligned}
$$

where $b_{1}, b_{2}, k_{1}$ and $k_{2}$ are the nonlinear parameters. Equation (1) is rewritten as

$$
\begin{aligned}
G_{0} b(x) u_{\mathrm{L}, \mathrm{C}}(t)= & a(t)+k(x) \omega_{0}^{2} x(t) \\
& +\left\{1+\left(1-\frac{Q_{0}}{Q_{\mathrm{m}}}\right)\left(b^{2}(x)-1\right)\right\} \frac{\omega_{0}}{Q_{0}} v(t),
\end{aligned}
$$

where

$$
\begin{aligned}
G_{0} & =\frac{B l_{0} A_{0}}{R_{\mathrm{dc}} m_{0}}, & \omega_{0} & =\sqrt{\frac{K_{0}}{m_{0}}}, \\
Q_{0} & =\frac{\sqrt{m_{0} K_{0}}}{R_{\mathrm{m}}+B l_{0}^{2} / R_{\mathrm{dc}}}, & Q_{m} & =\frac{\sqrt{m_{0} K_{0}}}{R_{\mathrm{m}}},
\end{aligned}
$$

$\omega_{0}=2 \pi f_{0}$ is the lowest resonance angular frequency $[\mathrm{rad} / \mathrm{s}]$, $f_{0}$ is the lowest resonance frequency $[\mathrm{Hz}], Q_{0}$ is the sharpness at $f_{0}$ and $Q_{\mathrm{m}}$ is the mechanical sharpness.

The goal of this study is to reduce the nonlinear distortions of an electrodynamic loudspeaker. If the nonlinear distortions 
are completely eliminated, then no distortions exist in the motion of the diaphragm, whose velocity is proportional to the sound pressure. This situation can be represented by the Eq. (4) with $b_{1}=b_{2}=k_{1}=k_{2}=0$. In this situation, $x(t), v(t)$ and $a(t)$ are expressed by

$$
\begin{aligned}
& x(t)=\mathcal{L}^{-1}\left[G_{0} /\left(s^{2}+\frac{\omega_{0}}{Q_{0}} s+\omega_{0}^{2}\right)\right] * u(t), \\
& v(t)=\mathcal{L}^{-1}\left[G_{0} s /\left(s^{2}+\frac{\omega_{0}}{Q_{0}} s+\omega_{0}^{2}\right)\right] * u(t), \\
& a(t)=\mathcal{L}^{-1}\left[G_{0} s^{2} /\left(s^{2}+\frac{\omega_{0}}{Q_{0}} s+\omega_{0}^{2}\right)\right] * u(t),
\end{aligned}
$$

where $\mathcal{L}^{-1}$ represents the inverse Laplace transform operator. In this case, $u_{\mathrm{L}, \mathrm{C}}(t)$ in Eq. (4) is a signal that can be used to reduce nonlinear distortions, which is called the compensation signal. Using a bilinear transform, the compensation signal at a discrete-time domain can be obtained as

$$
\begin{gathered}
u_{\mathrm{L}, \mathrm{C}}(n)=\frac{1}{b(x(n))} \mathcal{Z}^{-1}\left[H_{\mathrm{NL}}(z)\right] * u(n), \\
H_{\mathrm{NL}}(z)=\frac{C(x(n))+D(x(n)) z^{-1}+E(x(n)) z^{-2}}{1+B_{1, \mathrm{C}} z^{-1}+B_{2, \mathrm{C}} z^{-2}}, \\
x(n)=G_{0} \mathcal{Z}^{-1}\left[\frac{h_{x 0, \mathrm{C}}+h_{x 1, \mathrm{C}} z^{-1}+h_{x 2, \mathrm{C}} z^{-2}}{1+B_{1, \mathrm{C}} z^{-1}+B_{2, \mathrm{C}} z^{-2}}\right] * u(n),
\end{gathered}
$$

where $\mathcal{Z}^{-1}$ represents the inverse $\mathrm{Z}$ transform operator,

$$
\begin{aligned}
& C(x(n))=1+\beta(x(n))+\gamma(x(n)), \\
& D(x(n))=B_{1, \mathrm{C}}+2 \gamma(x(n)), \\
& E(x(n))=B_{2, \mathrm{C}}-\beta(x(n))+\gamma(x(n)), \\
& \beta(x(n))=\beta_{0, \mathrm{C}}\left\{b^{2}(x(n))-1\right\}, \beta_{0, \mathrm{C}}=\frac{\omega_{0}}{2 Q_{0} f_{\mathrm{s}} \alpha_{\mathrm{C}}}\left(1-\frac{Q_{0}}{Q_{\mathrm{m}}}\right), \\
& \gamma(x(n))=\gamma_{0, \mathrm{C}}\{k(x(n))-1\}, \gamma_{0, \mathrm{C}}=\frac{\omega_{0}^{2}}{4 f_{\mathrm{s}}^{2} \alpha_{\mathrm{C}}}, \\
& h_{x 0, \mathrm{C}}=h_{x 2, \mathrm{C}}=\frac{h_{x 1, \mathrm{C}}}{2}=\frac{1}{4 f_{\mathrm{s}}^{2} \alpha_{C}}, \quad B_{1, \mathrm{C}}=\left(-2+\frac{\omega_{0}^{2}}{2 f_{\mathrm{s}}^{2}}\right) / \alpha_{\mathrm{C}}, \\
& B_{2, \mathrm{C}}=\left(1-\frac{\omega_{0}}{2 Q_{0} f_{\mathrm{s}}}+\frac{\omega_{0}^{2}}{4 f_{\mathrm{s}}^{2}}\right) / \alpha_{\mathrm{C}}, \quad \alpha_{\mathrm{C}}=1+\frac{\omega_{0}}{2 Q_{0} f_{\mathrm{s}}}+\frac{\omega_{0}^{2}}{4 f_{\mathrm{s}}^{2}},
\end{aligned}
$$

and $f_{\mathrm{s}}$ is the sampling frequency $[\mathrm{Hz}]$. The compensation signal is generated by the filter structure shown in Fig. 1.

\section{MODIFICATION OF 2ND-ORDER NONLINEAR IIR FILTER}

In this section, the modification of the 2nd-order nonlinear IIR filter is explained. First, the basic ideas are discussed.

1) Desired linear characteristics

The linear characteristics of the electrodynamic loudspeaker are determined by the sharpness $Q_{0}$ and the lowest resonance angular frequency $\omega_{0}$. The original values of $Q_{0}$ and $\omega_{0}$ are not desired. For example, a large $Q_{0}$ causes a large displacement and the generation of nonlinear distortions, and a large $\omega_{0}$ leads to a low sound pressure below $\omega_{0}$. It is preferable to replace $Q_{0}$ and $\omega_{0}$ with the desired values $Q_{0, D}$ and $\omega_{0, \mathrm{D}}$, respectively. The

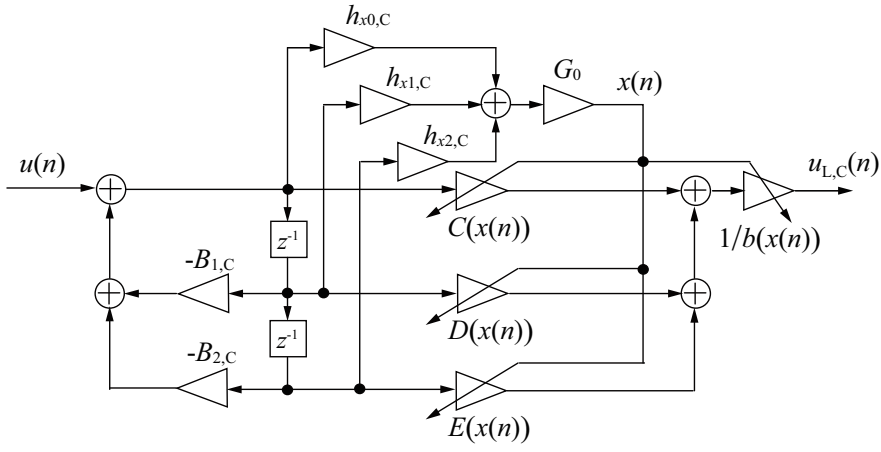

Fig. 1. Block diagram of 2nd-order nonlinear IIR filter.

linear characteristics with $Q_{0, D}$ and $\omega_{0, \mathrm{D}}$ are treated as the desired characteristics.

2) Nonlinear loudspeaker model

The nonlinear differential equation (1) or (4) can be used to simulate a real-world nonlinear loudspeaker. These equations have the original values of $\omega_{0}, Q_{0}$ and $Q_{\mathrm{m}}$ because these values for a real-world loudspeaker do not change before compensation. Therefore, the nonlinear loudspeaker model used to derive the compensation signal should include the original $\omega_{0}, Q_{0}$ and $Q_{\mathrm{m}}$.

Using the desired values $Q_{0, \mathrm{D}}$ and $\omega_{0, \mathrm{D}}$, the linear characteristics of the motion are rewritten as

$$
\begin{aligned}
& x_{\mathrm{D}}(t)=\mathcal{L}^{-1}\left[G_{0} /\left(s^{2}+\frac{\omega_{0, \mathrm{D}}}{Q_{0, \mathrm{D}}} s+\omega_{0, \mathrm{D}}^{2}\right)\right] * u(t), \\
& v_{\mathrm{D}}(t)=\mathcal{L}^{-1}\left[G_{0} s /\left(s^{2}+\frac{\omega_{0, \mathrm{D}}}{Q_{0, \mathrm{D}}} s+\omega_{0, \mathrm{D}}^{2}\right)\right] * u(t), \\
& a_{\mathrm{D}}(t)=\mathcal{L}^{-1}\left[G_{0} s^{2} /\left(s^{2}+\frac{\omega_{0, \mathrm{D}}}{Q_{0, \mathrm{D}}} s+\omega_{0, \mathrm{D}}^{2}\right)\right] * u(t) .
\end{aligned}
$$

Then, Eq. (4) is rewritten using $x_{\mathrm{D}}(t), v_{\mathrm{D}}(t)$ and $a_{\mathrm{D}}(t)$ as

$$
\begin{aligned}
G_{0} b\left(x_{\mathrm{D}}\right) u_{\mathrm{L}, \mathrm{M}}(t) & =a_{\mathrm{D}}(t)+k\left(x_{\mathrm{D}}\right) \omega_{0}^{2} x_{\mathrm{D}}(t) \\
& +\left\{1+\left(1-\frac{Q_{0}}{Q_{\mathrm{m}}}\right)\left(b^{2}\left(x_{\mathrm{D}}\right)-1\right)\right\} \frac{\omega_{0}}{Q_{0}} v_{\mathrm{D}}(t) .
\end{aligned}
$$

Similarly to the procedure for the conventional filter, the modified compensation signal $u_{\mathrm{L}, \mathrm{M}}(t)$ is derived by substituting Eqs. (11)-(13) into Eq. (14) at a discrete time domain.

Here, the linear component of $u_{\mathrm{L}, \mathrm{M}}(t)$ is focused on to show the principle of the compensation of $Q_{0}$ and $\omega_{0}$. The linear component of $u_{\mathrm{L}, \mathrm{M}}(t)$ is expressed as follows:

$$
\begin{aligned}
u_{\mathrm{L}, 1}(t) & =\frac{a_{\mathrm{D}}(t)}{G_{0}}+\frac{\omega_{0}}{Q_{0}} \frac{v_{\mathrm{D}}(t)}{G_{0}}+\omega_{0}^{2} \frac{x_{\mathrm{D}}(t)}{G_{0}} \\
& =\mathcal{L}^{-1}\left[\frac{s^{2}+\frac{\omega_{0}}{Q_{0}} s+\omega_{0}^{2}}{s^{2}+\frac{\omega_{0, \mathrm{D}}}{Q_{0, \mathrm{D}}} s+\omega_{0, \mathrm{D}}^{2}}\right] * u(t) .
\end{aligned}
$$

The linear characteristics of a real-world loudspeaker are simulated by Eqs. (5)-(7). Hence, by substituting Eq. (15) into Eqs. (5)-(7), the compensated linear characteristics can 
be obtained, for example, the displacement is compensated as follows:

$$
\begin{aligned}
x_{\text {real }}(t) & =\mathcal{L}^{-1}\left[\frac{G_{0}}{s^{2}+\frac{\omega_{0}}{Q_{0}} s+\omega_{0}^{2}}\right] * u_{\mathrm{L}, 1}(t) \\
& =\mathcal{L}^{-1}\left[\frac{G_{0}}{s^{2}+\frac{\omega_{0}}{Q_{0}} s+\omega_{0}^{2}} \times \frac{s^{2}+\frac{\omega_{0}}{Q_{0}} s+\omega_{0}^{2}}{s^{2}+\frac{\omega_{0, \mathrm{D}}}{Q_{0, \mathrm{D}}} s+\omega_{0, \mathrm{D}}^{2}}\right] * u(t) \\
& =\mathcal{L}^{-1}\left[\frac{G_{0}}{s^{2}+\frac{\omega_{0, \mathrm{D}}}{Q_{0, \mathrm{D}}} s+\omega_{0, \mathrm{D}}^{2}}\right] * u(t) .
\end{aligned}
$$

Thus, the linear characteristics, i.e, $Q_{0}$ and $\omega_{0}$, are compensated by $u_{\mathrm{L}, 1}(t) . v(t)$ and $a(t)$ are also compensated similarly to $x(t)$.

The modified compensation signal $u_{\mathrm{L}, \mathrm{M}}(n)$ can be derived using the desired linear characteristics of the motion (11)-(13) and the nonlinear differential equation (14) at a discrete time domain as follows:

$$
\begin{aligned}
& u_{\mathrm{L}, \mathrm{M}}(n)=\frac{1}{b\left(x_{\mathrm{D}}(n)\right)} \mathcal{Z}^{-1}\left[H_{\mathrm{NL}, \mathrm{M}}(z)\right] * u(n), \\
& H_{\mathrm{NL}, \mathrm{M}}(z)=\frac{C_{\mathrm{M}}\left(x_{\mathrm{D}}(n)\right)+D_{\mathrm{M}}\left(x_{\mathrm{D}}(n)\right) z^{-1}+E_{\mathrm{M}}\left(x_{\mathrm{D}}(n)\right) z^{-2}}{1+B_{1, \mathrm{M}} z^{-1}+B_{2, \mathrm{M}} z^{-2}}, \\
& x_{\mathrm{D}}(n)=G_{0} \mathcal{Z}^{-1}\left[\frac{h_{x 0, \mathrm{M}}+h_{x 1, \mathrm{M}} z^{-1}+h_{x 2, \mathrm{M}} z^{-2}}{1+B_{1, \mathrm{M}} z^{-1}+B_{2, \mathrm{M}} z^{-2}}\right] * u(n), \\
& C_{\mathrm{M}}\left(x_{\mathrm{D}}(n)\right)=\frac{\alpha_{\mathrm{C}}}{\alpha_{\mathrm{M}}}+\beta_{\mathrm{M}}\left(x_{\mathrm{D}}(n)\right)+\gamma_{\mathrm{M}}\left(x_{\mathrm{D}}(n)\right), \\
& D_{\mathrm{M}}\left(x_{\mathrm{D}}(n)\right)=B_{1, \mathrm{M}} \frac{\alpha_{\mathrm{C}}}{\alpha_{\mathrm{M}}}+2 \gamma_{\mathrm{M}}\left(x_{\mathrm{D}}(n)\right), \\
& E_{\mathrm{M}}\left(x_{\mathrm{D}}(n)\right)=B_{2, \mathrm{M}} \frac{\alpha_{\mathrm{C}}}{\alpha_{\mathrm{M}}}-\beta_{\mathrm{M}}\left(x_{\mathrm{D}}(n)\right)+\gamma_{\mathrm{M}}\left(x_{\mathrm{D}}(n)\right) \text {, } \\
& \beta_{\mathrm{M}}\left(x_{\mathrm{D}}(n)\right)=\beta_{0, \mathrm{M}}\left\{b^{2}\left(x_{\mathrm{D}}(n)\right)-1\right\}, \\
& \beta_{0, \mathrm{M}}=\frac{\omega_{0}}{2 Q_{0} f_{\mathrm{s}} \alpha_{\mathrm{M}}}\left(1-\frac{Q_{0}}{Q_{\mathrm{m}}}\right) \text {, } \\
& \gamma_{\mathrm{M}}\left(x_{\mathrm{D}}(n)\right)=\gamma_{0, \mathrm{M}}\left\{k\left(x_{\mathrm{D}}(n)\right)-1\right\}, \quad \gamma_{0, \mathrm{M}}=\frac{\omega_{0}^{2}}{4 f_{\mathrm{S}}^{2} \alpha_{\mathrm{M}}}, \\
& h_{x 0, \mathrm{M}}=h_{x 2, \mathrm{M}}=\frac{h_{x 1, \mathrm{M}}}{2}=\frac{1}{4 f_{\mathrm{s}}^{2} \alpha_{\mathrm{M}}}, \\
& B_{1, \mathrm{M}}=\left(-2+\frac{\omega_{0, \mathrm{D}}^{2}}{2 f_{\mathrm{s}}^{2}}\right) / \alpha_{\mathrm{M}}, \\
& B_{2, \mathrm{M}}=\left(1-\frac{\omega_{0, \mathrm{D}}}{2 Q_{0, \mathrm{D}} f_{\mathrm{s}}}+\frac{\omega_{0, \mathrm{D}}^{2}}{4 f_{\mathrm{s}}^{2}}\right) / \alpha_{\mathrm{M}}, \\
& \alpha_{\mathrm{M}}=1+\frac{\omega_{0, \mathrm{D}}}{2 Q_{0, \mathrm{D}} f_{\mathrm{s}}}+\frac{\omega_{0, \mathrm{D}}^{2}}{4 f_{\mathrm{S}}^{2}} .
\end{aligned}
$$

This compensation signal is generated by the modified block diagram shown in Fig. 2.

\section{EXPERIMENTAL RESULTS}

In this section, some experimental results are shown to verify the compensation performance of the modified 2ndorder nonlinear IIR filter. The specifications of the target loudspeaker used in this experiment are shown in Table I.

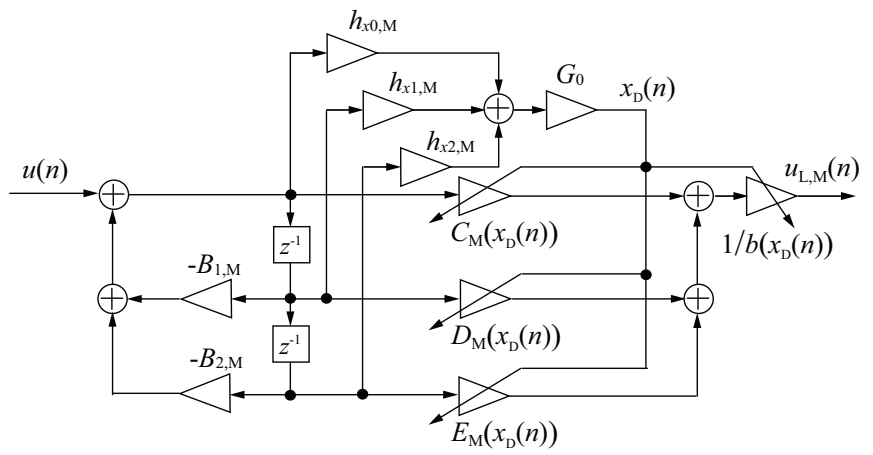

Fig. 2. Block diagram of modified 2nd-order nonlinear IIR filter.

TABLE I

SPECIFICATIONS OF THE TARGET LOUDSPEAKER.

\begin{tabular}{lr}
\hline Diameter & $6.5 \mathrm{~cm}$ \\
Rated input & $6 \mathrm{~W}(4.9 \mathrm{~V})$ \\
Nominal impedance & $4 \Omega$ \\
Enclosure type & Closed-box \\
Enclosure volume & $0.6 l$ \\
\hline
\end{tabular}

TABLE II

MEASURED LINEAR AND NONLINEAR PARAMETERS OF THE TARGET LOUDSPEAKER.

\begin{tabular}{lrrrr}
\hline$\omega_{0}$ & $1052 \mathrm{rad} / \mathrm{s}$ & & $K_{0}$ & $3480 \mathrm{~N} / \mathrm{m}$ \\
$Q_{0}$ & 1.78 & & $B l_{0}$ & $2.26 \mathrm{~Wb} / \mathrm{m}$ \\
$Q_{\mathrm{m}}$ & 5.32 & & $b_{1}$ & $-108 / \mathrm{m}$ \\
$R_{\mathrm{dc}}$ & $4.13 \Omega$ & & $b_{2}$ & $-78800 / \mathrm{m}^{2}$ \\
$R_{\mathrm{m}}$ & $0.62 \mathrm{Ns} / \mathrm{m}$ & & $k_{1}$ & $-38 / \mathrm{m}$ \\
$m_{0}$ & $3.14 \times 10^{-3} \mathrm{~kg}$ & & $k_{2}$ & $17100 / \mathrm{m}^{2}$ \\
\hline
\end{tabular}

\section{A. Measurement of the linear and nonlinear parameters}

Both the conventional and proposed 2nd-order nonlinear IIR filters require the linear and nonlinear parameters of the target loudspeaker. Measurements of these parameters were conducted using a measurement instrument (Klippel $\mathrm{GmbH}$ ) [7]. The measured linear and nonlinear parameters are shown in Table II. According to Table II, the sharpness of the loudspeaker $Q_{0}$ is large.

\section{B. Compensation experiments}

The nonlinear IIR filters were designed on the basis of measurement parameters in Table II. From this point forward, the conventional 2nd-order nonlinear IIR filter [4] and modified 2nd-order nonlinear IIR filter are referred to as the "conventional filter" and "proposed filter," respectively. In this experiment, $Q_{0, \mathrm{D}}$ and $\omega_{0, \mathrm{D}}$ were separately used to evaluate the effectiveness of the two filters separately. The arrangement of measurement equipments is shown in Table III, arrangement of measurement equipments is shown in Fig. 3 and the desired resonance frequency $f_{0, \mathrm{D}}$ is equal to $125 \mathrm{~Hz}$, lower than $f_{0}=167 \mathrm{~Hz}$. The signal length for each swept frequency is 1.28 seconds and the frame size for discrete Fourier transform is 2048 samples. The input voltage is set larger than the rated input because of generation of the large nonlinear distortions.

Firstly, the displacement of the diaphragm was measured by a laser displacement gauge with and without the proposed 
TABLE III

EXPERIMENTAL CONDITIONS

\begin{tabular}{lr}
\hline Input signal & Swept sinusoidal wave \\
Sampling frequency $f_{\mathrm{s}}$ & $32000 \mathrm{~Hz}$ \\
Fixed frequency $f_{1}$ & $188 \mathrm{~Hz}$ \\
Swept frequency $f_{2}$ & $30-4000 \mathrm{~Hz}$ \\
Input voltage & $4.5 \mathrm{Vrms}$ \\
Desired sharpness $Q_{0, \mathrm{D}}$ & 0.70 \\
Desired resonance angular frequency $\omega_{0, \mathrm{D}}$ & $785 \mathrm{rad} / \mathrm{s}$ \\
\hline
\end{tabular}

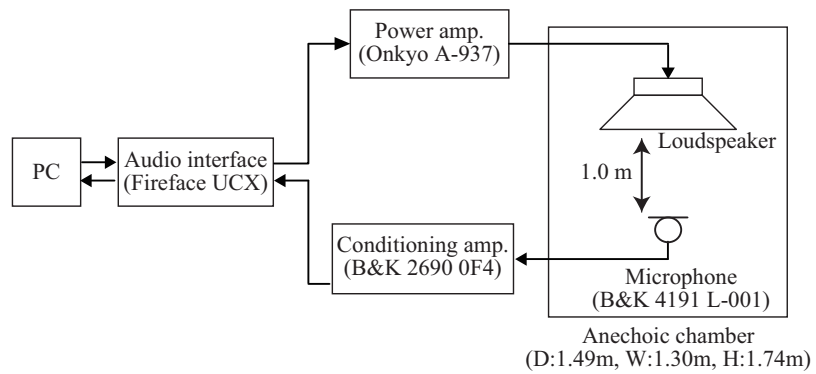

Fig. 3. Arrangement of measurement equipments.

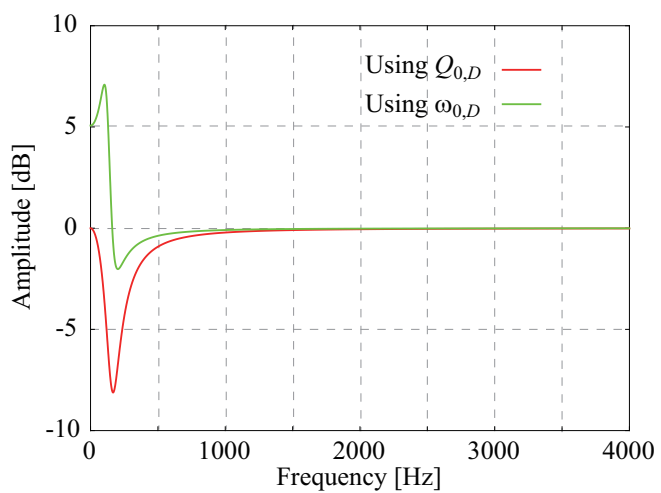

(a) Response of the proposed filter

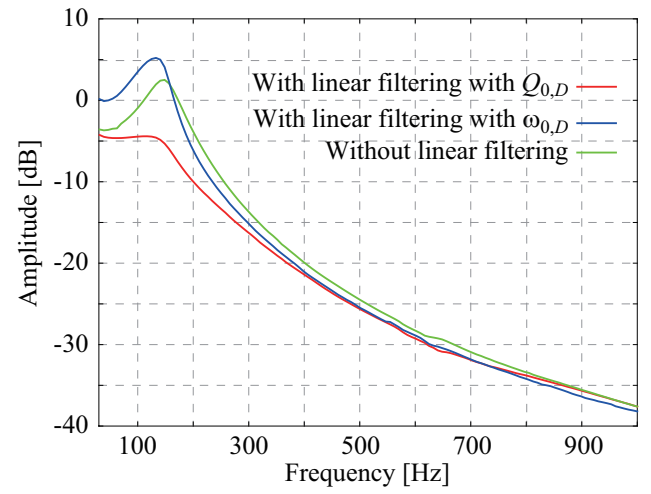

(b) Spectra of the measured displacement

Fig. 4. Results of compensating linear characteristic.

filters with $Q_{0, \mathrm{D}}$ and $\omega_{0, \mathrm{D}}$, respectively. The linear characteristics of the proposed filter and results of compensating the linear characteristic are shown in Fig. 4. According to Fig. 4, the linear characteristic was compensated by the proposed filter.

Next, the sound pressure of the nonlinear distortions was measured. The harmonic distortions were measured using a

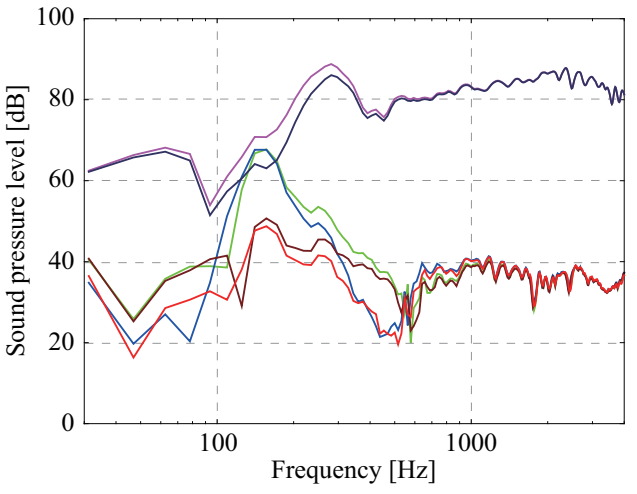

(a) Using the proposed filter with $Q_{0, \mathrm{D}}$

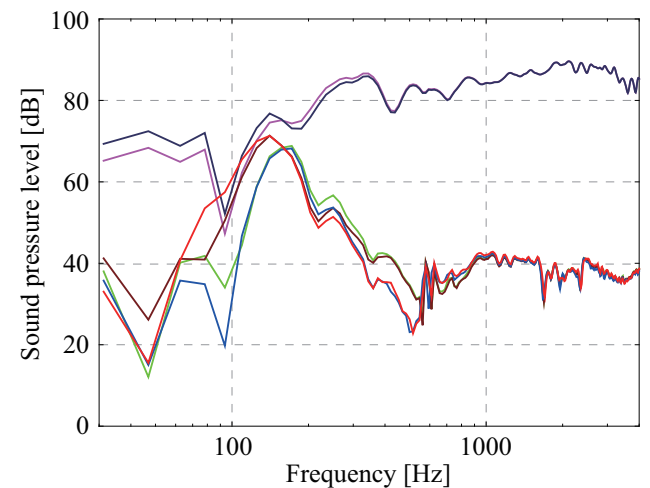

(b) Using the proposed filter with $\omega_{0, \mathrm{D}}$

- Fundamental component (proposed)

- Fundamental component (conventional)

- Before compensation

- After compensation (only linear filtering)

- After compensation (conventional)

- After compensation (proposed)

Fig. 5. Results of compensating harmonic distortions $\left(2 f_{2}\right)$.

single swept sinusoidal wave and the intermodulation distortions were measured using a multiple swept sinusoidal waves. The compensation signals were generated by the conventional and proposed filters. The measurement conditions are shown in Table III and the results after compensation are shown in Figs. 5-7. From Figs. 5-7, the proposed filter with a desired sharpness $Q_{0, \mathrm{D}}$ can compensate nonlinear distortions more effectively than the conventional filter. This is because the linear filtering by the proposed filter makes the displacement small around $f_{0}$, causing the nonlinear distortions to become small. On the other hand, the proposed filter with a desired resonance angular frequency $\omega_{0, \mathrm{D}}$ can compensate nonlinear distortions as effectively as the conventional filter above $f_{0}$, causing the nonlinear distortions to increase below $f_{0}$. This is because the proposed filter with $\omega_{0, \mathrm{D}}$ increases the displacement below $f_{0}$, causing the nonlinear distortions to increase. Hence, the proposed filter with $\omega_{0, \mathrm{D}}$ cannot improve the compensation performance.

Each results are indicated that the proposed and conventional filters cannot compensate the harmonic distortions above $600 \mathrm{~Hz}$. This is because these filters do not include the nonlinearity of the self-inductance. The compensation performance at high frequency will be improved by the same strategy, that is, including the nonlinearity of the self-inductance [8], [9]. 


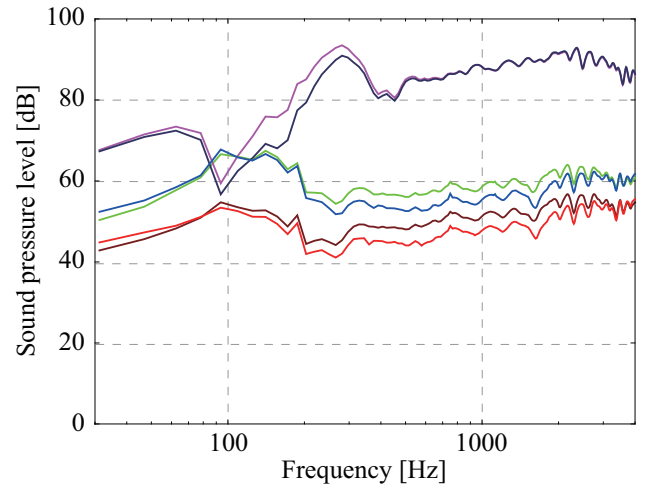

(a) Using the proposed filter with $Q_{0, \mathrm{D}}$

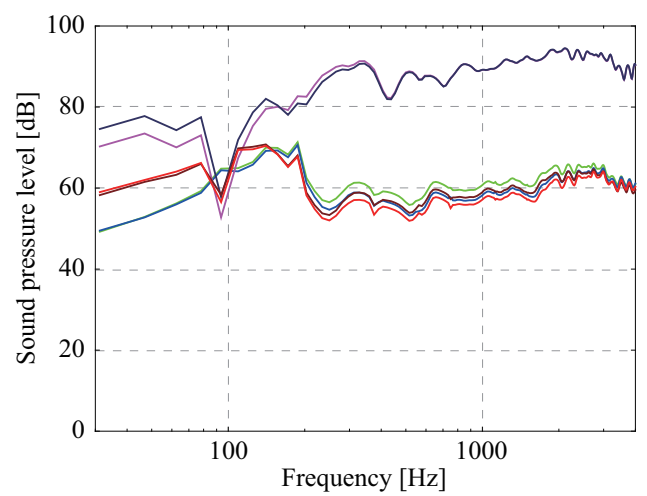

(b) Using the proposed filter with $\omega_{0, \mathrm{D}}$

- Fundamental component (proposed)

- Fundamental component (conventional)

- Before compensation

- After compensation (only linear filtering)

- After compensation (conventional)

- After compensation (proposed)

Fig. 6. Results of compensating intermodulation distortions $\left(f_{1}+f_{2}\right)$.

\section{CONClusion}

In this paper, a modified nonlinear IIR with a desired value of sharpness $Q_{0, \mathrm{D}}$ and a desired resonance angular frequency $\omega_{0, \mathrm{D}}$ was proposed to compensate linear and nonlinear distortions of electrodynamic loudspeakers. Experimental results demonstrated that the proposed filter with $Q_{0, \mathrm{D}}$ shows better performance than a conventional filter. However, the proposed filter with $\omega_{0, \mathrm{D}}$ cannot improve the compensation performance because it increases the displacement and nonlinear distortions.

In the future, we plan to modify the filter structure to improve the compensation performance. For example, focusing on high frequencies, the proposed filter with $Q_{0, \mathrm{D}}$ and that with $\omega_{0, \mathrm{D}}$ cannot compensate the harmonic distortions at high frequencies. This is because the proposed filter does not incorporate the effectiveness of self-inductance. We have already proposed a 3rd-order nonlinear IIR filter for improving the compensation performance at high frequencies [8], [9], which incorporates the effectiveness of self-inductance. Therefore, if the 3rd-order nonlinear IIR filter is modified similarly to the proposed 2nd-order nonlinear IIR filter, it is expected that the harmonic distortions at high frequencies can be reduced.

\section{ACKNOWLEDGMENT}

This work was supported by the MEXT-Supported Program for the Strategic Research Foundation at Private Universities,

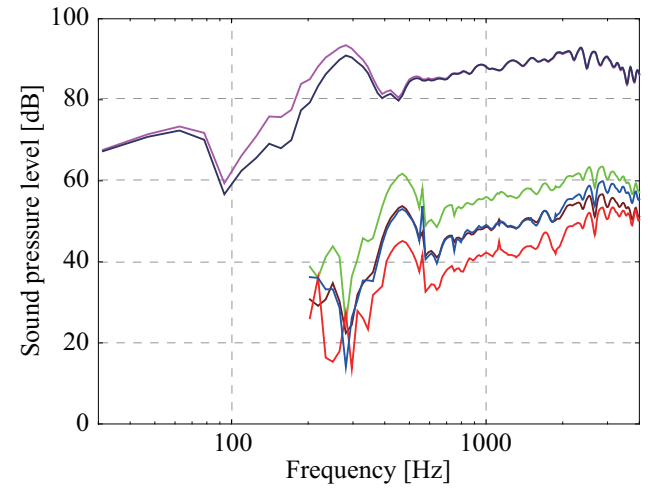

(a) Using the proposed filter with $Q_{0, \mathrm{D}}$

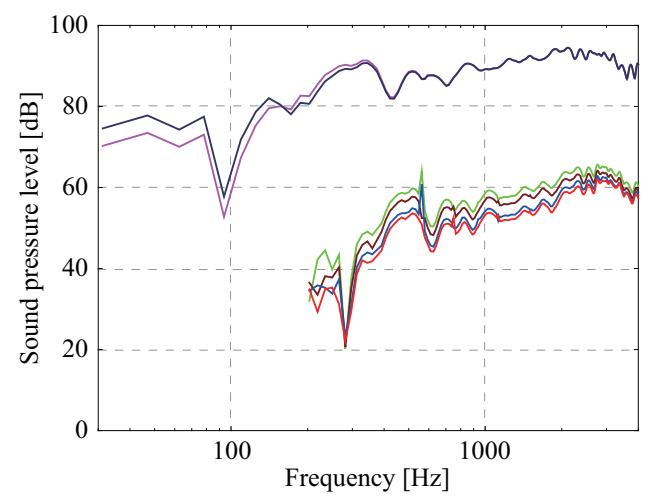

(b) Using the proposed filter with $\omega_{0, \mathrm{D}}$

- Fundamental component (proposed)

- Fundamental component (conventional)

- Before compensation

- After compensation (only linear filtering)

- After compensation (conventional)

- After compensation (proposed)

Fig. 7. Results of compensating intermodulation distortions $\left(f_{2}-f_{1}\right)$.

2013-2017.

\section{REFERENCES}

[1] W. Klippel, "Tutorial: Loudspeaker nonlinearities - causes, parameters, symptoms," Journal of the Audio Engineering Society, vol. 54, no. 10, pp. 907-939, Oct. 2006.

[2] W. Klippel, "The mirror filter-A new basis for reducing nonlinear distortion and equalizing response in woofer systems," Journal of the Audio Engineering Society, vol. 40, no. 9, pp. 675-691, Sep. 1992.

[3] H. Schurer, Linearization of Electroacoustic Transducers. University of Twente Publications, 1997.

[4] R. Nakao, Y. Kajikawa, and Y. Nomura, "An estimation method of parameters for closed-box loudspeaker system," IEICE Trans. on Fundamentals, vol. EA91-A, no. 10, pp. 3006-3013, Oct. 2008.

[5] K. Iwai and Y. Kajikawa, "A study on principle of compensating nonlinear distortions using 2nd-order nonlinear iir filter," in 2013 IEICE Society Conference, 2013.

[6] K. Iwai and Y. Kajikawa, "A study on principle of compensating nonlinear distortions using 3rd-order nonlinear iir filter," in 2015 IEICE General Conference, 2015.

[7] Klippel GmbH, "The development tool of audio technology," http://www. klippel.de/our-products/rd-system.html, 2013.

[8] K. Iwai and Y. Kajikawa, "Linearization of dynamic loudspeaker system using third-order nonlinear IIR filter," in 20th European Signal Processing Conference (EUSIPCO 2012), Aug. 2012, pp. 1970-1974, Bucharest, Romania.

[9] K. Iwai and Y. Kajikawa, "Third-order nonlinear IIR filter for compensating nonlinear distortions of loudspeaker systems," IEICE Trans. on Fundamentals, vol. E98-A, no. 3, pp. 820-832, Mar. 2015. 